\title{
The future of allogeneic hematopoietic stem cell transplantation: minimizing pain, maximizing gain
}

\author{
Marie-Térèse Little and Rainer Storb \\ Fred Hutchinson Cancer Research Center, Clinical Research Division, Transplantation Biology Program, Seattle, Washington 98109, USA \\ Address correspondence to: M.-T. Little, Fred Hutchinson Cancer Research Center, Clinical Research Division, \\ Transplantation Biology Program, 1100 Fairview Avenue, D1-100, PO Box 19024, Seattle, Washington 98109, USA. \\ Phone: (206) 667-4875; Fax: (206) 667-6124; E-mail: mlittle@fhcrc.org.
}

\begin{abstract}
Allogeneic hematopoietic stem cell transplantation (HSCT) has been used successfully to treat not only patients with hematological cancer but also patients with acquired marrow failure, genetic hematological diseases, and autoimmune diseases. It has also been much discussed as an adjunct or prelude to grafts of solid organs from the HSCT donor. Unfortunately, however, patients are prepared for HSCT by being blasted with high doses of chemotherapy or radiation therapy, treatments that have been thought necessary to suppress the patients' immune systems and to eradicate the underlying disease. Allografts then rescue the patients from the marrow aplasia induced by these harsh treatments. To better accomplish its aims, pretransplant therapy has been intensified to such a degree that toxicities to normal organs commonly result in morbidity or mortality, and therapyinduced pancytopenia sets the stage for
\end{abstract}

serious and even lethal infections. Given the toxicities, HSCT has been restricted to relatively young patients with good organ function, and the large majority of patients with candidate diseases are too old to experience the benefits of allografts. What is to be done about this state of affairs? Must HSCT operate by the maxim of "No pain, no gain"?

Several observations have raised doubts about the current approach to allogeneic HSCT. First, in many instances cancer cells cannot be eradicated entirely by high-dose cytotoxic therapy. Second, many of the observed cures have been brought about by immunological antitumor reactions generated by the allograft. Third, for many genetic diseases, a state of mixed donor-host chimerism appears to suffice to cure disease manifestations. These observations, combined with a better understanding of how to manipulate the immune system, have led to a radical rethinking of how allogeneic HSCT might employ less toxic strategies. For example, rather than trying to wipe out cancer cells through high-dose cytotoxic therapy, the HSCT donor's immune cells could be used for that purpose.

The article by Fuchimoto et al. in this issue of the JCI provides an excellent example of a novel allogeneic HSCT approach that avoids systemic toxicity by a high-dose preparatory regimen (1). Mixed donor-host hematopoietic chimerism was established in the swine model by in vivo depletion of recipient T cells with an anti-CD3 immunotoxin. In place of total-body irradiation (TBI), Fuchimoto et al. irradiated thymi of recipient animals directly, but they administered growth factor-mobilized peripheral blood cells to these animals at exceptionally high doses while suppressing endogenous immune responses with cyclosporine (CSP). Effective (> $90 \%$ ) in vivo T-cell depletion was accomplished with a pretransplant dose of a

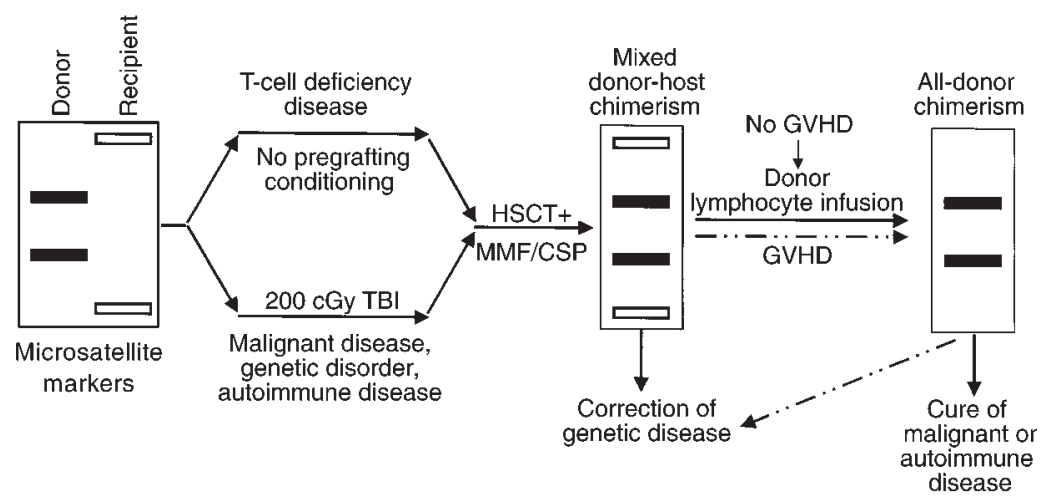

Figure 1

Schematic illustration of nonmyeloablative HSCT-induced mixed donor-host chimerism. Successful transplantation generally requires some level of host immunosuppression, except in cases of T-cell deficiency. This has often involved harsh TBI, but there is great interest in reducing the dosage to low, easily tolerated levels, such as the 200 cGy shown here, or even replacing this step entirely with other forms of conditioning. This process leads first to a state of mixed chimerism, in which both donor and recipient cells are detectable in the subject's lymphoid and myeloid compartments and thymus. To correct recessive genetic diseases, this state of mixed chimerism may suffice, whereas other conditions, such as malignancy and autoimmune disease, may require the complete replacement of host cells with donor cells. Such a state of all-donor chimerism can be initiated either in a spontaneous episode of graft-versus-host disease or through an infusion of donor lymphocytes. 
Diptheria toxin-anti-CD3 conjugate. Hematopoietic, liver, and kidney toxicities were minimal. Two pigs conditioned with $700 \mathrm{cGy}$ thymic irradiation and transplanted with $100 \times 10^{8}$ to 110 $\times 10^{8}$ swine leukocyte antigen-identical (SLA-identical) nucleated blood cells per kilogram developed low but stable mixed chimerism in both their lymphoid and their myeloid compartments. Thymic chimerism was lower but remained stable for over 300 days. Both animals rejected third-party skin grafts, and only one accepted the donor skin graft. The authors then applied the same conditioning regimen to two animals in an SLA-haploidentical setting (1). The first recipient animal developed low to undetectable levels of chimerism and eventually rejected the graft, while the second achieved higher levels of early chimerism, which decreased progressively by day 300 . Thus, the conditioning regimen permitted engraftment in the single haplotype-mismatched setting but failed to maintain a steady state of host/donor tolerance.

Reasoning that a further increase in thymic irradiation would be necessary to maintain engraftment across an MHC barrier, Fuchimoto and colleagues performed two additional haploidentical transplants, employing 1000 cGy thymic irradiation (1). Both animals achieved stable mixed and multilineage chimerism, although gut graft-versus-host disease (GVHD) complications in one animal led to its demise by day 73 after transplant. The other pig sustained mixed chimerism for over 400 days. Tolerance was confirmed in the two haploidentical mixed chimeras by sustained acceptance of donor-matched kidney allografts without additional immunosuppression.

It might be argued that the present findings are of limited relevance to the clinical setting, given the partially inbred nature of the swine. However, the observations are supported by previous findings with randomly bred dogs, and this canine work has already been validated by successful translation into the clinic, in protocols perhaps not so accurately termed "mini-transplants" (3). Whereas Fuchimoto et al. (1) focused on pretransplant host immunosuppression with anti-CD3 toxin and thymic irradiation, our canine studies aimed to let the obligatory postgrafting immunosuppression do "double duty," not only controlling GVHD but also eliminating host-versus-graft (rejection) reactions
(2). A powerful postgrafting regimen involving the antimetabolite mycophenolate mofetil (MMF) and the T-cell activation blocker CSP - obviated the need for intensive pretransplant therapy in these studies. This approach allowed stable allografts to be established after a single sublethal dose of 200 cGy TBI in dogs (2) and in human subjects who were too old or medically infirm to undergo conventional HSCT (3) (See Figure 1). Toxicities were mild, and human transplants were, for the most part, carried out in the outpatient setting. The graft-versus-tumor effects exerted by the allografts have, in many cases, resulted in molecular remissions of the underlying diseases. Donors included not only siblings, but also unrelated human leukocyte antigenmatched (HLA-matched) volunteers. In dogs, stable allografts were also seen when a TBI dose of only 100 cGy was combined with costimulatory signal blockade through CTLA4Ig (4). Finally, successful allografts were established when pretransplant therapy was reduced to a single dose of $450 \mathrm{cGy}$ radiation directed at a limited region of the recipient's central lymph node chain (5). Donor cells were firmly established in unirradiated marrow within 6 weeks of transplant and later became established at lymph node sites.

\section{Functions of irradiation in successful HSCT}

These findings challenge the concept that marrow space needs to be created by cytotoxic agents for grafts to home. Moreover, successful engraftment occurred with this protocol using only $4 \times 10^{8}$ marrow cells per kilogram as the source of hematopoietic stem cells (HSCs), rather than the $100 \times 10^{8}$ to $200 \times 10^{8}$ multicytokine-stimulated peripheral mononuclear cells per kilogram used in the current swine study (1). While such "mega-dose" transplants undoubtedly have advantages for engraftment, their success may not be entirely due to mass action as postulated by the authors (1), but rather to creation of marrow space by increased numbers of allogeneic immune cells infused with the graft. Rather than deluge the system with massive amounts of donor HSCs in order to displace the host's hematopoiesis, our approach harnesses the potential of immune cells in the graft, particularly the alloreactive $T$ cells. Given sufficient immunosup- pression to control rejection, these cells can create marrow space through subclinical GVH reactions. In human patients with genetically decreased $\mathrm{T}$ cell function, stable marrow allografts have been established without any pretransplant therapy, using only posttransplant MMF and CSP treatments (6). In addition, our observations in the canine model that stable mixed donorhost chimerism can be converted to alldonor chimerism by infusing donor lymphocytes support the idea that GVH reactions are the most important determinant of allograft success (5).

Because only $30 \%$ of patients have HLA-matched siblings, it will be crucial to overcome barriers to engraftment with HLA-mismatched cells. Preliminary work such as that reported here helps to lay the groundwork for future studies. Whether the requirement for high-dose (1000 cGy) thymic irradiation seen in the swine model extends to other species is not certain, and the precise advantage of this treatment either for creating space in the thymus for exogenous cells or for eliminating thymic alloreactivity - is likewise unclear. In canine studies, long-term engraftment has been achieved in 10 of 12 dog leukocyte antigen-haploidentical (DLA-haploidentical) littermate recipients after preconditioning with a relatively low dose of $450 \mathrm{cGy}$ TBI and an antibody to CD44 (7). However, it was also possible to lower the TBI dose further, to $200 \mathrm{cGy}$ (with an extended postgrafting regimen of CSP), while still allowing each of six DLA-haploidentical dogs to be successfully engrafted, three of them as stable mixed chimeras. Furthermore, after conditioning with the drug fludarabine and this well tolerated dose of 200 cGy TBI, engraftment occurred in each of four unrelated human recipients who received donor grafts that were mismatched at one HLA allele (8).

Thus, the era of "no pain, no gain" in HSCT may be coming to a close, as novel nonmyeloablative transplant strategies are being developed that offer successful engraftment with limited toxicity. The preclinical swine and canine studies have demonstrated the feasibility of relatively nontoxic approaches to HSCT, and these regimens have been translated successfully to human patients with malignant and nonmalignant diseases. Nevertheless, there is a need for more study in this area to fully understand how the 
immune system can be harnessed to promote graft survival, to prevent GVHD, and to maximize the graft-versus-tumor effect. The challenge in the future will be to develop clever strategies for allogeneic HSCT that obviate the need for even lowdose pregrafting irradiation.

\section{Acknowledgments}

This work was supported by grants from the National Institutes of Health (CA-78902, CA-18221, CA-15704, and HL-36444) and the Lady Tata Memorial Trust.
1. Fuchimoto, Y., et al. 2000. Mixed chimerism and tolerance without whole body irradiation in a large animal model. J. Clin. Invest. 105:1779-1789.

2. Storb, R., et al. 1997. Stable mixed hematopoietic chimerism in DLA-identical littermate dogs given sublethal total body irradiation before and pharmacological immunosuppression after marrow transplantation. Blood. 89:3048-3054.

3. McSweeney, P.A., et al. 1999. Outpatient PBSC allografts using immunosuppression with low-dose TBI before, and cyclosporine (CSP) and mycophenolate mofetil (MMF) after transplant. Blood. 94(Suppl. 1):519a. (Abstr.)

4. Storb, R., et al. 1999. Stable mixed chimerism in dogs given donor antigen, CTLA4Ig, and 100 cGy total body irradiation before and pharmacologic immunosuppression after marrow transplant Blood. 94:2523-2529.
5. Storb, R., et al. 1999. Stable mixed hematopoietic chimerism in dog leukocyte antigen-identical littermate dogs given lymph node irradiation before and pharmacologic immunosuppression after marrow transplantation. Blood. 94:1131-1136.

6. Woolfrey, A.E., et al. 1998. Non-myeloablative transplant regimen used for induction of multi-lineage allogeneic hematopoietic mixed donor-host chimerism in patients with T-cell immunodeficiency. Blood. 92(Suppl. 1):520a. (Abstr.)

7. Sandmaier, B.M., Yu, C., Gooley, T., Santos, E.B., and Storb, R. 1999. Haploidentical stem cell allografts after nonmyeloablative therapy in a preclinical large animal model. Blood. 94(Suppl. 1):318a. (Abstr.)

8. Hegenbart, U., et al. 2000. Hematopoietic stem cell transplants (HSCT) from HLA-mismatched unrelated donors after minimal conditioning. Bone Marrow Transplant. 25(Suppl. 1):S29. (Abstr.) 